\title{
WEIGHTED ESTIMATES ON FRACTAL DOMAINS
}

\section{RAFFAELA CAPITANELLI AND MARIA AGOSTINA VIVALDI}

\section{Dedicated to Professor V. G. Maz'ya}

Abstract. The aim of the paper is to establish estimates in weighted Sobolev spaces for the solutions of the Dirichlet problems on snowflake domains, as well as uniform estimates for the solutions of the Dirichlet problems on pre-fractal approximating domains.

$\S 1$. Introduction. In a previous paper [5], we established uniform estimates in weighted Sobolev spaces for the solutions of the Dirichlet problems on polygonal domains approximating the snowflake domain. In the present article, we deduce from the aforementioned estimates a regularity result for the Dirichlet problem on snowflake domain $\Omega_{3}$. More precisely, we prove that the second derivatives of the solution belong to the weighted space $L^{2}\left(\Omega_{3}, \delta^{\mu}\right), \mu>\mu^{*}=$ $\frac{2}{3}+\frac{1}{6} \log _{3} 4$, where by $\delta=\delta(x)$ we denote the distance of the point $x$ to the boundary $\partial \Omega_{3}$ of $\Omega_{3}$ (see Theorem 3.1). These results were presented by one of the authors at the meeting organized at the Department of Mathematical Sciences of the University of Liverpool in honour of Professor V. Maz'ya on the occasion of his 75th birthday. We are deeply grateful to Professor V. Maz'ya for having highlighted, in this meeting, Brennan's conjecture. Indeed, the regularity result of Theorem 3.1 could be greatly improved if Brennan's conjecture were shown to be true. As far as we know, Brennan's conjecture remains elusive, with only partial results having been established. In $\S 4$, we consider a larger class of fractal domains of the snowflake type constructed by means of the generalized Koch curves $K_{\alpha}, \alpha \in(2,4)$ (see $\$ 2$ ) and we prove, using the upper bound found by Hedenmalm and Shimorin [10], that the first derivatives of the solution to the Dirichlet problem on snowflake domain $\Omega_{\alpha}$ belong to the space $L^{q}\left(\Omega_{\alpha}\right)$, and the second derivatives belong to the weighted space $L^{2}\left(\Omega_{\alpha}, \delta^{\mu}\right)$, $\mu>\mu_{\alpha}=(2 / q)+((q-2) / 2 q) \log _{\alpha} 4, q<q_{0}=3.752$ (see Theorem 4.1). We note that the exponent $\mu^{*}$ is strictly greater than $\mu_{3}$ and the following inclusion holds for the weighted spaces $L^{2}\left(\Omega_{\alpha}, \delta^{\mu^{\prime}}\right) \subset L^{2}\left(\Omega_{\alpha}, \delta^{\mu^{\prime \prime}}\right)$ if $\mu^{\prime}<\mu^{\prime \prime}$. Hence, Theorem 4.1 improves Theorem 3.1 also for the case $\alpha=3$. In $\S 5$, by combining the tools and methods of the paper [5] with the results of $\S 4$, we establish uniform estimates that are more accurate than those established in [5], (compare Theorems 5.1 and 3.2). Lastly, in $\$ 6$ we briefly discuss how we can extend the results of previous sections to the solutions of obstacle problems. 
§2. Snowflakes. We recall the definition of the Koch curve with endpoints $A=(0,0)$ and $B=(1,0)$. We consider the family $\Psi^{\alpha}=\left\{\psi_{1}^{\alpha}, \ldots, \psi_{4}^{\alpha}\right\}$ of contractive similitudes $\psi_{i}^{\alpha}: \mathbb{C} \rightarrow \mathbb{C}, i=1, \ldots, 4$ with contraction factor $\alpha^{-1}$, $2<\alpha<4$ :

$$
\begin{gathered}
\psi_{1}^{\alpha}(z)=\frac{z}{\alpha}, \quad \psi_{2}^{\alpha}(z)=\frac{z}{\alpha} \mathrm{e}^{\mathrm{i} \theta(\alpha)}+\frac{1}{\alpha}, \\
\psi_{3}^{\alpha}(z)=\frac{z}{\alpha} \mathrm{e}^{-\mathrm{i} \theta(\alpha)}+\frac{1}{2}+\mathrm{i} \sqrt{\frac{1}{\alpha}-\frac{1}{4}}, \quad \psi_{4}^{\alpha}(z)=\frac{z-1}{\alpha}+1,
\end{gathered}
$$

where $\theta(\alpha)=\arcsin (\sqrt{\alpha(4-\alpha)} / 2)$.

By the general theory of self-similar fractals (see [12]), there exists a unique closed bounded set $K_{\alpha}$ which is invariant with respect to $\Psi^{\alpha}$, that is,

$$
K_{\alpha}=\bigcup_{i=1}^{4} \psi_{i}^{\alpha}\left(K_{\alpha}\right)
$$

Moreover, the Hausdorff dimension of the set $K_{\alpha}$ is $d_{f}=\ln _{\alpha} 4$. Let $K^{0}$ be the line segment of unit length that has as endpoints $A=(0,0)$ and $B=(1,0)$. We set, for each $n$ in $\mathbb{N}$,

$$
K_{\alpha}^{1}=\bigcup_{i=1}^{4} \psi_{i}^{\alpha}\left(K^{0}\right), \quad K_{\alpha}^{2}=\bigcup_{i=1}^{4} \psi_{i}^{\alpha}\left(K_{\alpha}^{1}\right), \ldots, K_{\alpha}^{n+1}=\bigcup_{i=1}^{4} \psi_{i}^{\alpha}\left(K_{\alpha}^{n}\right) ;
$$

$K_{\alpha}^{n}$ is the so-called $n$th pre-fractal curve. Moreover, the iterates $K_{\alpha}^{n}$ converge to the self-similar set $K_{\alpha}$ in the Hausdorff metric when $n$ tends to infinity (see [12]). Let $\Omega^{0}$ be the triangle with vertices $A=(0,0), B=(1,0)$, and $C=\left(\frac{1}{2},-\frac{\sqrt{3}}{2}\right)$. We construct on the side with endpoints $A$ and $B$ the pre-fractal Koch curve defined before, which will be denoted by $K_{1, \alpha}^{n}$, and the Koch curve defined before, which will be denoted by $K_{1, \alpha}$. In a similar way, we construct on the other sides the analogous pre-fractal Koch curves (the Koch curves) denoting by $K_{2, \alpha}^{n}$ and $K_{3, \alpha}^{n}$ (by $K_{2, \alpha}$ and $K_{3, \alpha}$ ) the curves with endpoints $B$ and $C$, and $C$ and $A$, respectively. We denote by $\Omega_{\alpha}^{n}$ the pre-fractal domain that is the set bounded by the pre-fractal Koch curves $K_{j, \alpha}^{n}, j=1,2,3$. Moreover, we denote by $\Omega_{\alpha}$ the snowflake that is the set bounded by the Koch curves $K_{j, \alpha}, j=1,2,3$. We consider the homogeneous Dirichlet problem in the snowflake domain $\Omega_{\alpha}$ as well as in the pre-fractal domains $\Omega_{\alpha}^{n}$ :

$$
\begin{aligned}
& \begin{cases}-\Delta u=f & \text { in } \Omega_{\alpha}, \\
u=0 & \text { on } \partial \Omega_{\alpha},\end{cases} \\
& \begin{cases}-\Delta u_{n}=f & \text { in } \Omega_{\alpha}^{n}, \\
u_{n}=0 & \text { on } \partial \Omega_{\alpha}^{n} .\end{cases}
\end{aligned}
$$

As is well known for any datum $f \in L^{2}\left(\Omega_{\alpha}\right)$, there exists a unique solution $u \in$ $H_{0}^{1}\left(\Omega_{\alpha}\right)$ of (2.3) by Lax-Milgram theorem. Analogously for any $f \in L^{2}\left(\Omega_{\alpha}^{n}\right)$ 
there exists a unique solution $u_{n} \in H_{0}^{1}\left(\Omega_{\alpha}^{n}\right)$ of (2.4) and the estimate

$$
\left.\left\|u_{n}\right\|\right|_{H_{0}^{1}\left(\Omega_{\alpha}^{n}\right)} \leqslant c\|f\|_{L^{2}\left(\Omega_{\alpha}^{n}\right)}
$$

holds with the constant $c$ independent of $n$. The previous estimate (2.5) follows from the following Poincaré-type inequality where the relevant fact is that the constant $C_{P}$ is independent of $n$.

PROPOSITION 2.1. There exists a constant $C_{P}$ independent of $n$ such that

$$
\|u\|_{L^{2}\left(\Omega_{\alpha}^{n}\right)} \leqslant C_{P}\left(\|\nabla u\|_{L^{2}\left(\Omega_{\alpha}^{n}\right)}+\|u\|_{L^{2}\left(\partial \Omega_{\alpha}^{n}\right)}\right)
$$

for all $u \in H^{1}\left(\Omega_{\alpha}^{n}\right)$.

The proof can be achieved as in [4, Theorem 7.3] by making some natural changes.

In the following sections we establish regularity results in weighted Sobolev spaces for the Dirichlet problem on snowflake domain $\Omega_{\alpha}$ and uniform estimates (in weighted Sobolev spaces) for the solutions of the Dirichlet problems on polygonal domains approximating the snowflake domain. From now on, by $c$ we denote (possibly different) positive constants independent of $n$.

§3. First regularity result. We introduce the weighted Lebesgue space $L^{2}\left(\Omega_{\alpha}, \delta^{\mu}\right)$, where by $\delta=\delta(x)$ we denote the distance of the point $x$ to the boundary $\partial \Omega_{\alpha}$ of $\Omega_{\alpha}$. The space $L^{2}\left(\Omega_{\alpha}, \delta^{\mu}\right)$ is the completion of the space $C^{0}\left(\bar{\Omega}_{\alpha}\right)$ with respect to the norm

$$
\|u\|_{L^{2}\left(\Omega_{\alpha}, \delta^{\mu}\right)}=\left(\int_{\Omega_{\alpha}}|u|^{2} \delta^{2 \mu} d x\right)^{1 / 2} .
$$

In this section, we choose $\alpha=3$ (and hence $\theta=\pi / 3$ ); in this case, the selfsimilar fractal $K_{3}$ is the so-called equilateral Koch curve and we prove the following theorem.

THEOREM 3.1. Let $u \in H_{0}^{1}\left(\Omega_{3}\right)$ be the solution of (2.3) with $f \in L^{2}\left(\Omega_{3}\right)$. Then, for every $\mu>\mu^{*}=\frac{2}{3}+\frac{1}{6} \log _{3} 4$,

$$
\sum_{|\beta|=2} \int_{\Omega_{3}}\left|D^{\beta} u\right|^{2} \delta^{2 \mu} d x \leqslant c\|f\|_{L^{2}\left(\Omega_{3}\right)}^{2} .
$$

In order to prove Theorem 3.1, we consider the solutions of the Dirichlet problems on polygonal domains $\Omega_{3}^{n}$ approximating the snowflake domain $\Omega_{3}$ and we use the uniform estimates in weighted Sobolev spaces established in [5]. We recall that as the domain $\Omega_{3}^{n}$ is not convex, then the solution $u_{n}$ of problem (2.4) does not belong to the space $H^{2}\left(\Omega_{3}^{n}\right)$. The second derivatives $\left|D^{\beta} u_{n}\right|\left(\beta=\left(\beta_{1}, \beta_{2}\right),|\beta|=2\right)$ actually belong to the weighted Lebesgue space $L^{2}\left(\Omega_{3}^{n}, \rho_{n}^{\mu}\right)$ for some positive exponent $\mu$. Here $\rho_{n}=\rho_{n}(x)$ denotes the distance 
function from the set $\mathcal{R}_{n}$ of the vertices of re-entrant corners of $\Omega_{3}^{n}$, and $L^{2}\left(\Omega_{3}^{n}\right.$, $\left.\rho_{n}^{\mu}\right)$ is the completion of the spaces $C^{0}\left(\overline{\Omega_{3}^{n}}\right)$ with respect to the norm

$$
\|u\|_{L^{2}\left(\Omega_{3}^{n}, \rho_{n}^{\mu}\right)}=\left(\int_{\Omega_{3}^{n}}|u|^{2} \rho_{n}^{2 \mu} d x\right)^{1 / 2} .
$$

In our setting, the domains $\Omega_{3}^{n}$ are polygonal, non-convex, and with an increasing number of corners, where the amplitude of the re-entrant corners is equal to $\frac{4}{3} \pi$. Hence, the celebrated Kondratiev result implies that

$$
\sum_{|\beta|=2} \int_{\Omega_{3}^{n}}\left|D^{\beta} u_{n}\right|^{2} \rho_{n}^{2 \mu} d x \leqslant c(n) \int_{\Omega_{3}^{n}} f^{2} \rho_{n}^{2 \mu} d x
$$

with $\mu \in\left(\frac{1}{4}, 1\right)$, (see $\left.[8,14]\right)$.

As the boundaries are the union of an increasing number of graphs and develop at the limit a fractal geometry, then the sharp regularity result (3.4) involves constants that might diverge as the number of graphs becomes infinite. In [5] we proved that there exists a suitable value of $\mu^{*}$ depending on the structural parameter of the limit fractal domain $\Omega_{3}$ for which uniform weighted estimates hold. More precisely, the following result holds.

THEOREM 3.2. Let $u_{n} \in H_{0}^{1}\left(\Omega_{3}^{n}\right)$ be the solution of (2.4) with $f \in L^{2}\left(\Omega_{3}^{n}\right)$. Then, for every $\mu>\mu^{*}=\frac{2}{3}+\frac{1}{6} \log _{3} 4$,

$$
\sum_{|\beta|=2} \int_{\Omega_{3}^{n}}\left|D^{\beta} u_{n}\right|^{2} \rho_{n}^{2 \mu} d x \leqslant c\|f\|_{L^{2}\left(\Omega_{3}^{n}\right)}^{2}
$$

with the constant $c$ independent of $n$.

Here, as before, $\rho_{n}$ denotes the distance function from the set $\mathcal{R}_{n}$ of the vertices of re-entrant corners of $\Omega_{3}^{n}$. It is well known that the solution $u_{n}$ of the Dirichlet problem (2.4) realizes the minimum of the following functional $F^{n}$ in $L^{2}\left(\Omega_{3}\right)$ :

$$
F^{n}[u]= \begin{cases}\int_{\Omega_{3}^{n}}|\nabla u|^{2} d x-2 \int_{\Omega_{3}^{n}} f u d x & \text { if }\left.u\right|_{\Omega_{3}^{n}} \in H_{0}^{1}\left(\Omega_{3}^{n}\right) \\ +\infty & \text { otherwise in } L^{2}\left(\Omega_{3}\right)\end{cases}
$$

It is easy to prove that the sequence of the functionals $F^{n}$ M-converges to the functional

$$
F[u]= \begin{cases}\int_{\Omega_{3}}|\nabla u|^{2} d x-2 \int_{\Omega_{3}} f u d x & \text { if } u \in H_{0}^{1}\left(\Omega_{3}\right), \\ +\infty & \text { otherwise in } L^{2}\left(\Omega_{3}\right),\end{cases}
$$

(see $[15,16]$ for definition and properties). 
From now on, we denote by the same symbol $u_{n}$ the extensions of the functions $u_{n}$ to zero outside $\Omega_{3}^{n}$. As a consequence of M-convergence we deduce that the sequence of the function $u_{n}$ strongly converges in $H_{0}^{1}\left(\Omega_{3}\right)$ to the function $u$ that minimizes the functional $F[u]$ defined in (3.7), and $u$ is the solution of (2.3).

We are now in a position to prove Theorem 3.1.

Proof. For any fixed $\mathrm{N}$, we consider regular open sets $G_{m}$ approximating $\Omega_{3}^{N}$, that is, $\bar{G}_{m} \subset G_{m+1} \uparrow \Omega_{3}^{N}, \bar{G}_{m} \subset \Omega_{3}^{N}$. We have, for any $n \geqslant N$, $D^{2} u_{n} \in L^{2}\left(G_{m}\right)$ and $D^{2} u \in L^{2}\left(G_{m}\right)$. We start by proving that, for a fixed $m$, the sequence $D^{2} u_{n}$ weakly converges to $D^{2} u$ in $L^{2}\left(G_{m}\right)$. Indeed, for $x \in G_{m}$, we have that, for any $n \geqslant N$,

$$
\rho_{n}(x) \geqslant \delta_{m, N}
$$

where $\delta_{m, N}$ denotes the distance of the set $G_{m}$ from the boundary $\partial \Omega_{3}^{N}$ of $\Omega_{3}^{N}$. From (3.5) we obtain

$$
\int_{G_{m}}\left|D^{2} u_{n}\right|^{2} d x \leqslant \delta_{m, N}^{-2 \mu} \int_{\Omega_{3}^{n}}\left|D^{2} u_{n}\right|^{2} \rho_{n}^{2 \mu} d x \leqslant c,
$$

with $c$ independent of $n$.

Then, there exists $w \in L^{2}\left(G_{m}\right)$ such that, up to passing to a subsequence, $D^{2} u_{n}$ weakly converges to $w$ in $L^{2}\left(G_{m}\right)$. Now we prove that

$$
w=D^{2} u \quad \text { almost everywhere in } G_{m},
$$

that is,

$$
\int_{G_{m}}\left(w-D^{2} u\right) \varphi d x=0
$$

for any $\varphi \in C_{0}^{1}\left(G_{m}\right)$. In fact,

$$
\begin{aligned}
& \int_{G_{m}}\left(w-D^{2} u\right) \varphi d x \\
& \quad=\int_{G_{m}} w \varphi d x+\int_{G_{m}} D u D \varphi d x=\int_{G_{m}} w \varphi d x+\lim _{n} \int_{G_{m}} D u_{n} D \varphi d x \\
& =\int_{G_{m}} w \varphi d x-\lim _{n} \int_{G_{m}} D^{2} u_{n} \varphi d x=0 .
\end{aligned}
$$

From (3.9) we deduce that (for any fixed $m$ ) the sequence $D^{2} u_{n}$ weakly converges to $D^{2} u$ in $L^{2}\left(G_{m}, \delta^{\mu}\right)$, that is,

$$
\int_{G_{m}}\left(D^{2} u_{n}-D^{2} u\right) \varphi \delta^{2 \mu} d x \rightarrow 0
$$

for any $\varphi \in L^{2}\left(G_{m}\right)$. We show that $D^{2} u \in L^{2}\left(\Omega, \delta^{\mu}\right)$. We recall that $\delta=\delta(x)$ denotes the distance of the point $x$ to the boundary $\partial \Omega_{\alpha}$ of $\Omega_{\alpha}$. We set, for 
$n \geqslant N, w_{m}^{n}=\chi_{G_{m}} D^{2} u_{n}$, where by $\chi_{G_{m}}$ we denote the indicatrix function of the set $G_{m}$. Then we have that $\lim _{m} w_{m}^{n}=D^{2} u_{n}$ almost everywhere in $\Omega_{3}^{N}$. Hence,

$$
\begin{aligned}
\int_{\Omega_{3}^{N}}\left|D^{2} u\right|^{2} \chi_{G_{m}} \delta^{2 \mu} d x & =\int_{G_{m}}\left|D^{2} u\right|^{2} \delta^{2 \mu} d x \\
& \leqslant \liminf \int_{G_{m}}\left|D^{2} u_{n}\right|^{2} \delta^{2 \mu} d x \\
& \leqslant \liminf \int_{\Omega_{3}^{n}}\left|D^{2} u_{n}\right|^{2} \rho_{n}^{2 \mu} d x \leqslant c .
\end{aligned}
$$

As $\lim _{m}\left|D^{2} u\right|^{2} \chi_{G_{m}} d^{2 \mu}=\left|D^{2} u\right| \delta^{2 \mu}$ almost everywhere in $\Omega_{3}^{N}$, we obtain, by Fatou's lemma,

$$
\int_{\Omega_{3}^{N}}\left|D^{2} u\right|^{2} \delta^{2 \mu} d x \leqslant \liminf \int_{\Omega_{3}^{N}}\left|D^{2} u\right|^{2} \chi_{G_{m}} \delta^{2 \mu} d x \leqslant c .
$$

Finally, as the sets $\Omega_{3}^{N}$ tend to $\Omega_{3}$ analogously, we have

$$
\int_{\Omega_{3}}\left|D^{2} u\right|^{2} \delta^{2 \mu} d x \leqslant c
$$

This concludes the proof of Theorem 3.1, and §3.

$\S 4$. Brennan's conjecture. We point out that estimate (3.2) (with the same exponent $\mu^{*}$ ) was established by Nyström in the more general framework of the class Domain $\left(2, M, r_{0}, q\right)$. We recall that an open, connected, and bounded subset $D$ of $\mathbb{R}^{2}$ belongs to the class Domain $\left(2, M, r_{0}, q\right)$ if $D$ is a nontangentially accessible (NTA) domain with parameters $M$ and $r_{0}$, supporting the reverse Hölder inequality (see (4.1) below). We say that a set $D$ supports a reverse Hölder inequality if for all $P \in \partial D, r<r_{0}$,

$$
J\left(P, r, D, x_{0}, q\right) \leqslant C J\left(P, r, D, x_{0}, 1\right),
$$

where

$$
J\left(P, r, D, x_{0}, a\right)=\left(\frac{1}{|B(P, r) \cap D|} \int_{B(P, r) \cap D}\left|\frac{G(x)}{\operatorname{distance}(x, \partial D)}\right|^{a} d x\right)^{1 / a}
$$

for $a \in[1, \infty)$. Here $B(P, r)$ denotes an open ball, centred at $P$ and of radius $r$, and $G(x)=G\left(x, x_{0}\right)$ the Green function of $D$ with fixed pole $x_{0}$, such that the quotient of distance $\left(x_{0}, \partial D\right)$ and diameter $(D)$ is bounded from above and below by absolute constants. By $|B(P, r) \cap D|$ we denote the 2-dimensional Lebesgue measure of the set. We now recall the definition of NTA domains (see [13]).

Definition 4.1. A bounded domain $D \subset \mathbb{R}^{2}$ is an NTA domain when there exist constants $M$ and $r_{0}>0$ such that: 
(i) Corkscrew condition. For any $P \in \partial D, r<r_{0}$, there exists $A=A_{r}(P) \in$ $D$ such that $M^{-1} r<|A-P|<r$ and $\operatorname{dist}(A, \partial D)>M^{-1} r$;

(ii) $D^{c}$ satisfies the corkscrew condition (where $D^{c}$ denotes the complement of $D)$; and

(iii) Harnack chain condition. If $\varepsilon>0, P_{1}$ and $P_{2}$ belong to $D, \operatorname{dist}\left(P_{j}, \partial D\right)>$ $\varepsilon$, and $\left|P_{1}-P_{2}\right|<C \varepsilon$, then there exists a sequence of $M$-non-tangential balls $\left(B(A, r)\right.$ is an $M$-non-tangential ball if $M^{-1} r<\operatorname{dist}(B(A, r)$, дD) $<M r$ ) such that the first ball contains $P_{1}$, the last contains $P_{2}$, and such that consecutive balls have a non-empty intersection, whose length depends on $C$, but not $\varepsilon$.

We stress the fact that estimate (3.2) could be greatly improved if Brennan's conjecture were shown to be true.

More precisely, Brennan (see [2, Theorem 1]) proved that

$$
\int_{D}\left|\phi^{\prime}\right|^{q} d x<+\infty \text { for all } q, 4 / 3<q<q_{0}
$$

where the upper bound $q_{0}$ is strictly larger than three. Here, $D$ denotes a simply connected domain in $\mathbb{R}^{2}$ with at least two boundary points and $\phi$ is a conformal map to the open disk B. Moreover, Brennan postulated that $q_{0}=4$ was indeed the correct upper bound for all domains $D$.

In our setting, we choose $D=\Omega_{\alpha}$, where $\Omega_{\alpha}$ is the fractal domain of the snowflake type constructed by means of the generalized Koch curves $K_{\alpha}, \alpha \in(2$, 4) (see §2). Hence, if Brennan's conjecture were shown to be true, we could prove that the second derivatives of the solution to the Dirichlet problem on snowflake domain $\Omega_{\alpha}$ belong to the weighted space $L^{2}\left(\Omega_{\alpha}, d^{\mu_{\alpha}}\right), \mu_{\alpha}>\mu_{B, \alpha}=$ $\frac{1}{2}+\frac{1}{4} \log _{\alpha} 4$. We note that the exponent $\mu^{*}$ is strictly greater than $\mu_{B, 3}$ and the following inclusion holds for the weighted spaces $L^{2}\left(\Omega_{\alpha}, \delta^{\mu^{\prime}}\right) \subset L^{2}\left(\Omega_{\alpha}, \delta^{\mu^{\prime \prime}}\right)$ if $\mu^{\prime}<\mu^{\prime \prime}$.

Unfortunately, as far as we know, Brennan's conjecture remains elusive, and there are only partial results (see e.g. [7, 11]). More precisely, the upper bound for which (4.2) is known to hold has been increased by Pommerenke [19] to $q_{0}=3.399$ and by Hedenmalm and Shimorin [10] to $q_{0}=3.752$. We use the result of Hedenmalm and Shimorin to improve the results of Theorem 3.1.

THEOREM 4.1. Let $u \in H_{0}^{1}\left(\Omega_{\alpha}\right)$ be the solution of (2.3) with $f \in L^{2}\left(\Omega_{\alpha}\right)$. Then, for every $\mu_{\alpha}>\mu_{P, \alpha}=\left(2 / q_{0}\right)+\left(\left(q_{0}-2\right) / 2 q_{0}\right) \log _{\alpha} 4, q_{0}=3.752$,

$$
\sum_{|\beta|=2} \int_{\Omega_{\alpha}}\left|D^{\beta} u\right|^{2} \delta^{2 \mu_{\alpha}} d x \leqslant c\|f\|_{L^{2}\left(\Omega_{\alpha}\right)}^{2} .
$$

We note that the exponent $\mu^{*}$ is strictly greater than $\mu_{P, 3}$, hence Theorem 4.1 improves Theorem 3.1 also for the case $\alpha=3$.

In order to prove Theorem 4.1, we need to introduce notation and preliminaries. An important key tool is the Whitney decomposition $W_{\Omega_{\alpha}}$ of the snowflake $\Omega_{\alpha}$ by means of closed cubes whose sides are parallel to a fixed 
system of coordinate axes. By $x_{Q}$ we mean the centre of the cube $Q$ and by $H Q$, $H>0$, the cube $Q$ dilated with respect to $x_{Q}$ by a factor $H$; by $\ell(Q)$ we mean the side length of $Q$ (see, for instance, [20]). The decomposition $W_{\Omega_{\alpha}}$ of $\Omega_{\alpha}$ has the following properties:

(a) $\Omega_{\alpha}=\bigcup_{j=1}^{+\infty} Q_{j}$;

(b) $Q_{j}^{\circ} \cap Q_{k}^{\circ}=\emptyset$ if $j \neq k$; and

(c) there exist constants $C_{1}$ and $C_{2}$ such that

$$
C_{1} \ell\left(Q_{j}\right) \leqslant \text { distance }\left(Q_{j}, \partial \Omega \alpha\right) \leqslant C_{2} \ell\left(Q_{j}\right)
$$

Associated with the decomposition, we consider cut functions $\phi_{j} \in C^{\infty}\left(\mathbb{R}^{2}\right)$ such that:

(d) $\phi_{j}=1$ on $Q_{j}$;

$$
\begin{gathered}
\operatorname{supp} \phi_{j} \subset H Q_{j} \quad \text { with } 1<H<C_{1}+1 ; \\
\left|D^{\beta} \phi_{j}\right| \leqslant \frac{c(H)}{\ell\left(Q_{j}\right)^{|\beta|}} ; \text { and }
\end{gathered}
$$

(g) Locally finite covering condition. For any fixed $j,\left(\operatorname{supp} \phi_{j}\right)^{\circ} \cap\left(\operatorname{supp} \phi_{k}\right)^{\circ}$ $\neq \emptyset$ only for a number $M_{0}$ (depending on $H$ but not on $j$ ) of indices $k$.

Proposition 4.1. Let $u \in H_{0}^{1}\left(\Omega_{\alpha}\right)$ be the solution of (2.3) with $f \in L^{2}\left(\Omega_{\alpha}\right.$, $\left.\delta^{\mu}\right)$. Then, for every $\mu>0$,

$$
\begin{aligned}
& \sum_{|\beta|=2} \int_{\Omega_{\alpha}}\left|D^{\beta} u\right|^{2} \delta^{2 \mu} d x \\
& \quad \leqslant c\left(\int_{\Omega_{\alpha}} f^{2} \delta^{2 \mu} d x+\int_{\Omega_{\alpha}}|\nabla u|^{2} \delta^{2 \mu-2} d x+\int_{\Omega_{\alpha}} u^{2} \delta^{2 \mu-4} d x\right) .
\end{aligned}
$$

Proof. We extend the functions $u$ and $f$ to zero outside $\Omega_{\alpha}$ denoting them by the same symbols. By using the decomposition of the domain $\Omega_{\alpha}$ we obtain

$$
\int_{\Omega_{\alpha}}\left|D^{\beta} u\right|^{2} \delta^{2 \mu} d x=\sum_{j=1}^{+\infty} \int_{Q_{j}}\left|D^{\beta} u\right|^{2} \delta^{2 \mu} d x .
$$

We consider $u_{j}=u \phi_{j}$; then we have

$$
\begin{cases}-\Delta u_{j}=\chi_{H Q_{j}} f_{j} & \text { in } H Q_{j} \\ u=0 & \text { on } \partial H Q_{j}\end{cases}
$$

where $f_{j}=f \phi_{j}-2 \nabla u \nabla \phi_{j}-u \Delta \phi_{j}$. By the classical results [6, 20] and also [8],

$$
\sum_{|\beta|=2} \int_{H Q_{j}}\left|D^{\beta} u_{j}\right|^{2} d x \leqslant c \int_{H Q_{j}}\left|\Delta u_{j}\right|^{2} d x,
$$


we obtain with the properties (4.6) and (4.4)

$$
\begin{aligned}
\sum_{|\beta|=2} \int_{Q_{j}}\left|D^{\beta} u\right|^{2} d x & =\sum_{|\beta|=2} \int_{Q_{j}}\left|D^{\beta} u_{j}\right|^{2} d x \\
& \leqslant c\left(\int_{H Q_{j}} f^{2} d x+\int_{H Q_{j}}|\nabla u|^{2} \delta^{-2} d x+\int_{H Q_{j}} u^{2} \delta^{-4} d x\right) .
\end{aligned}
$$

By multiplying (4.10) by $\ell_{j}^{2 \mu}$ where $\ell_{j}=\ell\left(Q_{j}\right)$ and by using (4.4) and (4.5) we obtain

$$
\begin{aligned}
& \sum_{|\beta|=2} \int_{Q_{j}}\left|D^{\beta} u\right|^{2} \delta^{2 \mu} d x \\
& \leqslant\left(C_{2}+1\right)^{2 \mu} \sum_{|\beta|=2} \int_{Q_{j}}\left|D^{\beta} u\right|^{2} \ell_{j}^{2 \mu} d x \\
& \quad \leqslant c\left(\int_{H Q_{j}} f^{2} \delta^{2 \mu} d x+\int_{H Q_{j}}|\nabla u|^{2} \delta^{2 \mu-2} d x+\int_{H Q_{j}} u^{2} \delta^{2 \mu-4} d x\right) .
\end{aligned}
$$

From (4.8), and (4.11), we obtain the inequality (4.7) for every $\mu>0$.

We evaluate the last term in estimate (4.7) by means of the Hardy inequality and we refer to [17, Theorem 5.1] for the proof (see also [22]).

Proposition 4.2. Let $u \in H_{0}^{1}\left(\Omega_{\alpha}\right)$; then there exists a constant $c$ such that, for $s<2-\log _{\alpha} 4$,

$$
\int_{\Omega_{\alpha}} u^{2} \delta^{s-2} d x \leqslant c \int_{\Omega_{\alpha}}|\nabla u|^{2} \delta^{s} d x .
$$

We can now prove Theorem 4.1.

Proof. From Proposition 4.1, we have that

$$
\begin{aligned}
& \sum_{|\beta|=2} \int_{\Omega_{\alpha}}\left|D^{\beta} u\right|^{2} \delta^{2 \mu} d x \\
& \quad \leqslant c\left(\int_{\Omega_{\alpha}} f^{2} \delta^{2 \mu} d x+\int_{\Omega_{\alpha}}|\nabla u|^{2} \delta^{2 \mu-2} d x+\int_{\Omega_{\alpha}} u^{2} \delta^{2 \mu-4} d x\right) .
\end{aligned}
$$

By Proposition 4.2 with $s=2 \mu-2$ we obtain

$$
\int_{\Omega_{\alpha}} u^{2} \delta^{2 \mu-4} d x \leqslant c \int_{\Omega_{\alpha}}|\nabla u|^{2} \delta^{2 \mu-2} d x .
$$

Hence, we only need to evaluate the $L^{q}$-norm of the gradient of the Green potential. At this point, we combine the estimate of the upper bound in Brennan's conjecture due to Hedenmalm and Shimorin (see [10]) with consequences for the 
Green potential shown by Hedenmalm (see [9, Theorem 4.2 and Corollary 4.5]) to obtain

$$
\left(\int_{\Omega_{\alpha}}|\nabla G f|^{q} d x\right)^{1 / q} \leqslant C(q)\left(\int_{\Omega_{\alpha}}|f|^{2} d x\right)^{1 / 2}
$$

with $2<q<q_{0}, q_{0}=3.752$.

We use estimate (4.13) and we have

$$
\begin{aligned}
\int_{\Omega_{\alpha}}|\nabla u|^{2} \delta^{2 \mu-2} d x & \leqslant\left(\int_{\Omega_{\alpha}}|\nabla u|^{q} d x\right)^{2 / q}\left(\int_{\Omega_{\alpha}} \delta^{(q /(q-2))(2 \mu-2)} d x\right)^{(q-2) / q} \\
& \leqslant c\left(\int_{\Omega_{\alpha}} f^{2} d x\right)\left(\int_{\Omega_{\alpha}} \delta^{(q /(q-2))(2 \mu-2)} d x\right)^{(q-2) / q} .
\end{aligned}
$$

Then $\int_{\Omega_{\alpha}} \delta^{(q /(q-2))(2 \mu-2)} d x$ is bounded as $(q /(q-2))(2 \mu-2)+2-d_{f}>0$ where $d_{f}=\log _{\alpha} 4$ is the Hausdorff dimension of $\partial \Omega_{\alpha}$. This concludes the proof of Theorem 4.1 and $\$ 4$.

§5. Uniform estimates. In this section, we establish uniform estimates for solutions of the homogeneous Dirichlet problem in the approximating domains $\Omega_{\alpha}^{n}$ that improve those established in [5] (compare Theorems 5.1 and 3.2). Our approach combines tools and methods of the paper [5] with the result of $\S 4$. In order to use the decomposition of the pre-fractal snowflake constructed in [5] we focus our attention only on the case $\alpha=3$ (and hence on $\Omega_{3}^{n}$ ). We could obviously construct a suitable decomposition for any pre-fractal snowflake $\Omega_{\alpha}^{n}$, but as doing this with appropriate details would require some extra work, we prefer to address this tool in a forthcoming article. Moreover, as long as we consider the same framework of the paper [5], we can skip the details and highlight the main differences, referring to [5] for the complete proofs.

More precisely, we state the following result.

THEOREM 5.1. Let $u_{n} \in H_{0}^{1}\left(\Omega_{3}^{n}\right)$ be the solution of (2.4) with $f \in L^{2}\left(\Omega_{3}^{n}\right)$. Then, for every $\mu>\mu_{P, 3}=\left(2 / q_{0}\right)+\left(\left(q_{0}-2\right) / 2 q_{0}\right) \log _{3} 4, q_{0}=3.752$,

$$
\sum_{|\beta|=2} \int_{\Omega_{3}^{n}}\left|D^{\beta} u_{n}\right|^{2} \rho_{n}^{2 \mu} d x \leqslant c\|f\|_{L^{2}\left(\Omega_{3}^{n}\right)}^{2}
$$

where the constant $c$ is independent of $n$.

We recall that $\rho_{n}=\rho_{n}(x)$ denotes the distance function from the set $\mathcal{R}_{n}$ of the vertices of re-entrant corners of $\Omega_{3}^{n}$.

Before proving Theorem 5.1, some remarks on the results of $\S 4$ are needed.

Remark 5.1. For a domain $D$ in the class of self-similar domains $\operatorname{SF}(q)$ (see [18, Definition 12.1]) the validity of estimate (4.13) is equivalent to boundedness of the term

$$
I_{q}(D)=\sum_{j \geqslant 4} 2^{j(q-2)} \sum_{Q \in W_{j}} G\left(x_{Q}\right)^{q}
$$


where $W_{j}:=\left\{Q \in W_{D}, \ell(Q)=2^{-j}\right\}, G(x)=G\left(x, x_{0}\right)$, denotes the Green function and distance $\left(x_{0}, \partial D\right) \sim \operatorname{diameter}(D)$. We recall that $x_{Q}$ means the centre of the cube $Q, \ell(Q)$ means the side length of $Q$, and $W_{D}$ denotes the Whitney decomposition of $D$ mentioned in $\S 4$. Moreover, for a domain $D \in S F(q)$ it is possible to rephrase the reverse Hölder inequality condition (4.1) in terms of the sum over the Whitney cubes (5.2) (see [18, §12]).

In conclusion, as any domain $\Omega_{3}$ belongs to the class $S F(q)$ for any $q>1$ (see [18, §12]), then from (4.13) we deduce that any domain $\Omega_{\alpha}$ supports the reverse Hölder inequality for any exponent $q<q_{0}=3.752$.

Remark 5.2. We stress the fact that any domain $\Omega_{\alpha}$ is an NTA domain (see e.g. [18]) and from Remark 5.1 we deduce that any domain $\Omega_{\alpha}$ belongs to the class Domain $\left(2, M, r_{0}, q\right)$ for any exponent $q<q_{0}=3.752$.

Remark 5.3. A peculiar property of the NTA domains is that if $D$ is an NTA domain with parameters $M, r_{0}$, then $D \in \operatorname{Domain}\left(2, M, r_{0}, 1+1 /(1-\beta)\right)$ where $\beta=\beta(M)>0$ is a constant describing the boundary behaviour of the Green function (see [18]). More precisely, the constant $\beta$ is the constant appearing in the inequality (see [18])

$$
G(x, y) \leqslant C(M) \frac{\operatorname{dist}(y, \partial D)^{\beta}}{r^{\beta}} w(x, B(Q, r) \cap \partial D, D)
$$

where $x \in D \backslash B\left(Q_{0}, C r\right), Q_{0} \in \partial D, C r<r_{0}$, and $y \in B\left(Q_{0}, r\right) \cap D$. Here $w(x, F, D)$ is the harmonic measure of $F \subset \partial D$ relative to $D$ at $x \in D$. From Remark 5.2, we deduce that $\Omega_{\alpha} \in \operatorname{Domain}\left(2, M, r_{0}, 1+1 /(1-\beta)\right)$ with $\beta=$ $(q-2) /(q-1)$. Moreover, the value of $\beta$ in (5.3) is the same as the one that appears in the following inequality (see [13, Lemma 4.1]):

$$
u(x)<c(M)\left(\frac{|x-P|}{r}\right)^{\beta} C(u),
$$

for all $P \in \partial D, r<r_{0}$, and for every positive harmonic function u in $D$ such that u vanishes continuously on $B(P, r) \cap \partial D$. Here $x \in B(Q, r) \cap D$ and $C(u):=$ $\sup \{u(y): y \in \partial B(Q, r) \cap D\}$.

We are now in a position to prove Theorem 5.1.

Proof. By proceeding as in the proof of [5, Theorem 4.1], we establish the estimate for every $\mu>\frac{1}{4}$ :

$$
\begin{aligned}
& \sum_{|\beta|=2} \int_{\Omega_{3}^{n}}\left|D^{\beta} u\right|^{2} \rho_{n}^{2 \mu} d x \\
& \quad \leqslant c\left(\int_{\Omega_{3}^{n}} f^{2} \rho_{n}^{2 \mu} d x+\int_{\Omega_{3}^{n}}|\nabla u|^{2} \rho_{n}^{2 \mu-2} d x+\int_{\Omega_{3}^{n}} u^{2} \rho_{n}^{2 \mu-4} d x\right)
\end{aligned}
$$


with the constant $c$ independent of $n$. We evaluate the last term in estimate (5.5) by means of the Hardy-type inequality established in [5, Theorem 5.1]:

$$
\int_{\Omega_{3}^{n}} u^{2} \rho_{n}^{s-2} d x \leqslant c \int_{\Omega_{3}^{n}}|\nabla u|^{2} \rho_{n}^{s} d x
$$

for any $u \in H_{0}^{1}\left(\Omega_{3}^{n}\right), s<2$, with the constant $c$ independent of $n$ and of $u$. Hence, we only need to establish uniform $L^{q}$-estimates for the gradient of the Green potential and we achieve this aim by using estimate (4.13) in $\$ 4$, the geometry of the approximating domains $\Omega_{3}^{n}$, a monotonicity argument, and the peculiar properties of a particular class of NTA domains mentioned in the previous remark (see also $[\mathbf{1 3}, \mathbf{1 8}]$ ). Indeed, Nyström proves that (see [18]) for any $D \in \operatorname{Domain}\left(2, M, r_{0}, q\right)$ where $q>2$ there exists a constant $C=C=C(M$, $\left.r_{0}, q\right)$ such that if $1 / q=1 / p-1 / 2$ then the following inequality is valid for all $f \in L^{p}(D)$ :

$$
\left(\int_{D}|\nabla G f|^{q} d x\right)^{1 / q} \leqslant C\left(M, r_{0}, q\right)\left(\int_{D}|f|^{p} d x\right)^{1 / p} .
$$

A peculiar fact is that the constant in (5.6) depends only on the parameters $M, r_{0}$, and $q$ (appearing in the definition of the class $\operatorname{Domain}\left(2, M, r_{0}, q\right)$ ). Therefore, in order to prove uniform bounds, we only have to show that all domains $\Omega_{3}^{n}$ belong to the class $\operatorname{Domain}\left(2, M, r_{0}, q\right)$ with the same values $M, r_{0}$ and $q$. In [3, Lemma 2.3], it is proved that all domains $\Omega_{3}^{n}$ satisfy the Ahlfors threepoint condition with the same Ahlfors constant $A$ (independent of $n$ ). From $[\mathbf{1}$, Lemma 2.5], we deduce that all domains $\Omega_{3}^{n}$ are NTA domains with the same constants $M$ and $r_{0}$ and from Remark 5.3, $\Omega_{3}^{n} \in \operatorname{Domain}\left(2, M, r_{0}, 1+1 /(1-\beta)\right)$ where $\beta=\beta(M)>0$ is the constant appearing in (5.4). We note that the value of $\beta$ in (5.4) can be expressed in terms of the capacity of the set $B(P$, $r) \cap\left(\Omega_{3}^{n}\right)^{c}$ (where $\left(\Omega_{3}^{n}\right)^{c}$ denotes the complement of $\Omega_{3}^{n}$ ). In our setting, the sequence of domains $\Omega_{3}^{n}$ increases and converges to the snowflake $\Omega_{3}$; moreover, the boundary of $\Omega_{3}$ contains all the vertices of $\Omega_{3}^{n}$. Then $B(P, r) \cap\left(\Omega_{3}^{n}\right)^{c} \supset B(P$, $r) \cap\left(\Omega_{3}\right)^{c}$ for any $P$ vertex of $\Omega_{3}^{n}$ and for any point $P^{*}$ of $\partial \Omega_{3}^{n} \backslash \mathcal{R}_{n}$ the capacity of the set $B(P, r) \cap\left(\Omega_{3}^{n}\right)^{c}$ is greater than or equal to the capacity of the set $B(P, r) \cap\left(\Omega_{3}^{n}\right)^{c}$ for some $P \in \mathcal{R}_{n}$. Hence, the value $\beta=\left(q_{0}-2\right) /\left(q_{0}-1\right)$, $q_{0}=3.752$ being a lower bound for the snowflake $\Omega_{3}$ (see Remarks 5.2 and 5.3), provides a uniform lower bound for $\Omega_{3}^{n}$. In conclusion,

$$
\begin{aligned}
\int_{\Omega_{3}^{n}}|\nabla u|^{2} \rho_{n}^{2 \mu-2} d x & \leqslant\left(\int_{\Omega_{3}^{n}}|\nabla u|^{q} d x\right)^{2 / q}\left(\int_{\Omega_{3}^{n}} \rho_{n}^{(q /(q-2))(2 \mu-2)} d x\right)^{(q-2) / q} \\
& \leqslant\left(\int_{\Omega_{3}^{n}} f^{2} d x\right)\left(\int_{\Omega_{3}^{n}} \rho_{n}^{(q /(q-2))(2 \mu-2)} d x\right)^{(q-2) / q}
\end{aligned}
$$

Finally, to show the uniform boundedness of the term $\int_{\Omega_{3}^{n}} \rho_{n}^{(q /(q-2))(2 \mu-2)} d x$, we note that for any $x \in \Omega_{3}^{n}$, we have that $\rho_{n}(x) \geqslant \delta(x)$ where $\delta(x)$ denotes the 
distance of the point $x$ from the boundary of $\Omega_{3}$. Then

$$
\begin{aligned}
\int_{\Omega_{3}^{n}} \rho_{n}^{(q /(q-2))(2 \mu-2)} d x & \leqslant \int_{\Omega_{3}^{n}} \delta^{(q /(q-2))(2 \mu-2)} d x \\
& \leqslant \int_{\Omega_{3}} \delta^{(q /(q-2))(2 \mu-2)} d x<+\infty
\end{aligned}
$$

as $(q /(q-2))(2 \mu-2)+2-d_{f}>0$ where $d_{f}=\log _{3} 4$ is the Hausdorff dimension of $\partial \Omega_{3}$. This completes the proof of Theorem 5.1 and $\S 5$.

§6. Obstacle problems. In this section, we briefly discuss how we can extend the results of the previous sections to the solutions of obstacle problems. In the notation of the previous section, we consider the problem: find a function $u \in \mathcal{K}$ such that

$$
\int_{\Omega_{\alpha}} \nabla u(\nabla u-\nabla v) d x \leqslant \int_{\Omega_{\alpha}} g(u-v) d x \quad \text { for all } v \in \mathcal{K}
$$

where $\mathcal{K}=\left\{v \in H_{0}^{1}\left(\Omega_{\alpha}\right): \varphi_{1} \leqslant u \leqslant \varphi_{2}\right\}, \varphi_{i} \in H^{1}\left(\Omega_{\alpha}\right), i=1,2, \Delta \varphi_{i} \in$ $L^{2}\left(\Omega_{\alpha}\right), i=1,2, \varphi_{1} \leqslant \varphi_{2}$ in $\Omega_{\alpha}$, and $\varphi_{1} \leqslant 0 \leqslant \varphi_{2}$ on $\partial \Omega_{\alpha}$.

Moreover, we consider the sequence of obstacle problems in the sets $\Omega_{\alpha}^{n}$ : find a function $u \in \mathcal{K}^{n}$ such that

$$
\int_{\Omega_{\alpha}^{n}} \nabla u(\nabla u-\nabla v) d x \leqslant \int_{\Omega_{\alpha}^{n}} g(u-v) d x \quad \text { for all } v \in \mathcal{K}^{n}
$$

where $\mathcal{K}^{n}=\left\{v \in H_{0}^{1}\left(\Omega_{\alpha}^{n}\right): \varphi_{1}^{n} \leqslant u \leqslant \varphi_{2}^{n}\right\}, \varphi_{i}^{n} \in H^{2}\left(\Omega_{\alpha}^{n}\right), \varphi_{1} \leqslant \varphi_{2}$ in $\Omega_{\alpha}^{n}$, and $\varphi_{1}^{n} \leqslant 0 \leqslant \varphi_{2}^{n}$ on $\partial \Omega_{\alpha}^{n}$.

As is well known for any datum $g \in L^{2}\left(\Omega_{\alpha}\right)$, there exists a unique solution $u \in H_{0}^{1}\left(\Omega_{\alpha}\right)$ of (6.1) by the Lax-Milgram theorem. Moreover, for any $g \in$ $L^{2}\left(\Omega_{\alpha}^{n}\right)$ there exists a unique solution $u_{n} \in H_{0}^{1}\left(\Omega_{\alpha}^{n}\right)$ of (6.2) by the Lax-Milgram theorem and the following estimate holds:

$$
\left.\left\|u_{n}\right\|\right|_{H_{0}^{1}\left(\Omega_{\alpha}^{n}\right)} \leqslant c\left(\|g\|_{L^{2}\left(\Omega_{\alpha}^{n}\right)}+\left\|\Delta \varphi_{1}^{n}\right\|_{L^{2}\left(\Omega_{\alpha}^{n}\right)}+\left\|\Delta \varphi_{2}^{n}\right\|_{L^{2}\left(\Omega_{\alpha}^{n}\right)}\right)
$$

with the constant $c$ independent of $n$.

We state the following results.

THEOREM 6.1. Using the previous notation and assumptions, let $u$ be the solution of (6.1) with $g \in L^{2}\left(\Omega_{\alpha}\right)$. Then, for every $\mu_{\alpha}>\mu_{P, \alpha}=\left(2 / q_{0}\right)+$ $\left(\left(q_{0}-2\right) / 2 q_{0}\right) \log _{\alpha} 4, q_{0}=3.752$,

$$
\sum_{|\beta|=2} \int_{\Omega_{\alpha}}\left|D^{\beta} u\right|^{2} \delta^{2 \mu_{\alpha}} d x \leqslant c\left(\|g\|_{L^{2}\left(\Omega_{\alpha}\right)}^{2}+\left\|\Delta \varphi_{1}\right\|_{L^{2}\left(\Omega_{\alpha}\right)}^{2}+\left\|\Delta \varphi_{2}\right\|_{L^{2}\left(\Omega_{\alpha}\right)}^{2}\right) .
$$


THEOREM 6.2. Using the previous notation and assumptions, let $u_{n}$ be the solution of (6.2) with $g \in L^{2}\left(\Omega_{3}\right)$ Then, for every $\mu>\mu_{P, 3}=\left(2 / q_{0}\right)+$ $\left(\left(q_{0}-2\right) / 2 q_{0}\right) \log _{3} 4, q_{0}=3.752$,

$$
\sum_{|\beta|=2} \int_{\Omega_{3}^{n}}\left|D^{\beta} u_{n}\right|^{2} \rho_{n}^{2 \mu} d x \leqslant c\left(\|g\|_{L^{2}\left(\Omega_{3}^{n}\right)}^{2}+\left\|\Delta \varphi_{1}^{n}\right\|_{L^{2}\left(\Omega_{3}^{n}\right)}^{2}+\left\|\Delta \varphi_{2}^{n}\right\|_{L^{2}\left(\Omega_{3}^{n}\right)}^{2}\right)
$$

where the constant $c$ is independent of $n$.

We recall that $\rho_{n}=\rho_{n}(x)$ denotes the distance function from the set $\mathcal{R}_{n}$ of the vertices of re-entrant corners of $\Omega_{3}^{n}$. The proofs follow from Theorems 4.1 and 5.1 by using the so-called Lewy-Stampacchia inequality (see [21, Theorem 4.35] and the reference quoted there). More precisely, we use the following.

PROPOSITION 6.1. In the previous assumptions the solution $u$ of (6.1) satisfies in $\Omega_{\alpha}$ the inequality

$$
\left(-\triangle \varphi_{2}\right) \wedge g \leqslant(-\triangle u) \leqslant\left(-\triangle \varphi_{1}\right) \vee g
$$

Analogously, the solution $u_{n}$ of (6.2) satisfies in $\Omega_{\alpha}^{n}$

$$
\left(-\triangle \varphi_{2}^{n}\right) \wedge g \leqslant\left(-\triangle u_{n}\right) \leqslant\left(-\triangle \varphi_{1}^{n}\right) \vee g
$$

Proof of Theorem 6.1. By Proposition 6.1 the solution $u$ of (6.1) is the solution of the Dirichlet problem (2.3) with datum $f \in L^{2}\left(\Omega_{\alpha}\right)$; in fact, from inequality (6.6) we obtain

$$
\left(-\triangle \varphi_{2}\right) \wedge g \leqslant f \leqslant\left(-\triangle \varphi_{1}\right) \vee g
$$

Using Theorem 4.1 we thus conclude the proof.

Proof of Theorem 6.2. By Proposition 6.1 the solution $u_{n}$ of (6.2) is the solution of the Dirichlet problem (2.4) with datum $f \in L^{2}\left(\Omega_{\alpha}^{n}\right)$; in fact from inequality (6.7) we obtain

$$
\left(-\triangle \varphi_{2}^{n}\right) \wedge g \leqslant f \leqslant\left(-\triangle \varphi_{1}^{n}\right) \vee g
$$

Using Theorem 5.1 we thus conclude the proof and $\S 6$.

Acknowledgements. We wish to thank Professor Alexander Movchan for his kind invitation and Professor V. G. Maz'ya for his valuable suggestions.

The authors are members of the Gruppo Nazionale per l'Analisi Matematica, la Probabilità e le loro Applicazioni (GNAMPA) of the Istituto Nazionale di Alta Matematica (INdAM). The authors are supported by Grant Ateneo "Sapienza" 2013. 


\section{References}

1. B. Bennewitz and J. L. Lewis, On the dimension of p-harmonic measure. Ann. Acad. Sci. Fenn. Math. 30(2) (2005), 459-505.

2. J. E. Brennan, The integrability of the derivative in conformal mapping. J. Lond. Math. Soc. 18(2) (1978), 261-272.

3. R. Capitanelli, Robin boundary condition on scale irregular fractals. Commun. Pure Appl. Anal. 9(5) (2010), 1221-1234; doi:10.3934/cpaa.2010.9.1221.

4. R. Capitanelli and M. A. Vivaldi, On the Laplacean transfer across fractal mixtures. Asymptot. Anal. 83(1-2) (2013), 1-33; doi:10.3233/ASY-2012-1149.

5. R. Capitanelli and M. A. Vivaldi, Uniform weighted estimates on pre-fractal domains. Discrete Contin. Dyn. Syst. Ser. B 19(7) (2014), 1969-1985; doi:10.3934/dcdsb.2014.19.1969.

6. D. Gilbarg and N. Trudinger, Elliptic Partial Differential Equations of Second Order (Grundlehren der Mathematischen Wissenschaften [Fundamental Principles of Mathematical Sciences] 224), 2nd edn edn., Springer (Berlin, 1983).

7. V. Gol'dshtein and A. Ukhlov, Brennan's Conjecture and universal Sobolev inequalities. Bull. Sci. Math. 138(2) (2014), 253-269.

8. P. Grisvard, Elliptic Problems in Non-Smooth Domains, Pitman (Boston, 1985).

9. H. Hedenmalm, The dual of a Bergman space on simply connected domains. J. Anal. Math. 88 (2002), 311-335.

10. H. Hedenmalm and S. Shimorin, Weighted Bergman spaces and the integral means spectrum of conformal mappings. Duke Math. J. 127(2) (2005), 341-393.

11. R. Hurri-Syrjänen and S. G. Staples, A quasiconformal analogue of Brennan's conjecture. Complex Var. Elliptic Equ. 35 (1998), 27-32.

12. J. E. Hutchinson, Fractals and selfsimilarity. Indiana Univ. Math. J. 30 (1981), 713-747.

13. D. S. Jerison and C. E. Kenig, Boundary behaviour of harmonic functions in non-tangentially accessible domains. Adv. Math. 46 (1982), 80-147.

14. V. A. Kondratiev, Boundary value problems for elliptic equations in domains with conical or angular points. Tr. Mosk. Mat. Obs. 16 (1967), 209-292.

15. U. Mosco, Convergence of convex sets and of solutions of variational inequalities. Adv. Math. 3 (1969), 510-585.

16. U. Mosco, Composite media and asymptotic Dirichlet forms. J. Funct. Anal. 123(2) (1994), 368-421.

17. K. Nyström, Smoothness properties of Dirichlet problems in domains with a fractal boundary. $P h D$ Dissertation, Umeà, 1994.

18. K. Nyström, Integrability of Green potentials in fractal domains. Ark. Mat. 34 (1996), 335-381.

19. Ch. Pommerenke, On the integral means of the derivative of a univalent function. J. Lond. Math. Soc. 32(2) (1985), 254-258.

20. E. M. Stein, Singular Integrals and Differentiability Properties of Functions (Princeton Mathematical Series 30), Princeton University Press (Princeton, NJ, 1970).

21. G. M. Troianiello, Elliptic Partial Differential Equations and Obstacle Problems (The University Series in Mathematics), Plenum Press (New York, 1987).

22. A. Wannebo, Hardy inequalities. Proc. Amer. Math. Soc. 109(1) (1990), 85-95.

Raffaela Capitanelli,

Dipartimento di Scienze di Base e Applicate per l'Ingegneria,

"Sapienza Università di Roma",

Via A. Scarpa 16, 00161 Roma,

Italy

E-mail: raffaela.capitanelli@uniroma1.it
Maria Agostina Vivaldi,

Dipartimento di Scienze di Base e

Applicate per l'Ingegneria,

"Sapienza Università di Roma",

Via A. Scarpa 16, 00161 Roma,

Italy

E-mail: maria.vivaldi@sbai.uniroma1.it 